\title{
La révolution batave à l'entrée du troisième millénaire. Nouveaux problèmes, nouvelles approches, nouveaux objets
}

Annie Jourdan et Joost Rosendaal

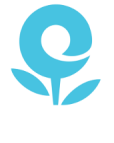

\section{OpenEdition}

Édition électronique

URL : https://journals.openedition.org/ahrf/460

DOI : $10.4000 /$ ahrf.460

ISSN : 1952-403X

Éditeur :

Armand Colin, Société des études robespierristes

Édition imprimée

Date de publication : 1 décembre 2001

Pagination : 1-23

ISSN : 0003-4436

Référence électronique

Annie Jourdan et Joost Rosendaal, « La révolution batave à l'entrée du troisième millénaire. Nouveaux problèmes, nouvelles approches, nouveaux objets », Annales historiques de la Révolution française [En ligne], 326 | octobre- décembre 2001, mis en ligne le 07 mars 2008, consulté le 23 avril 2022. URL: http://journals.openedition.org/ahrf/460 ; DOI : https://doi.org/10.4000/ahrf.460 


\title{
INTRODUCTION LA RÉVOLUTION BATAVE À L'ENTRÉE DU TROISIÈME MILLÉNAIRE NOUVEAUX PROBLÈMES, NOUVELLES APPROCHES, NOUVEAUX OBJETS
}

\author{
ANNIE JOURDAN \& JOOST ROSENDAAL
}

\begin{abstract}
Cette contribution fait le point sur l'historiographie de la période franco-batave (1795-1813), à partir des études et des thèses récentes. II s'agit de savoir quels sont leurs apports dans les divers domaines historiques; en quoi elles renouvellent les interprétations classiques et sous quelles perspectives ce renouvellement est envisageable. De là, une attention non seulement à la grande politique, mais aussi à celle des localités ou à celle que dévoilent les concepts. Outre les lois et les concepts qui trahissent des priorités quelquefois peu disertes, les rites, les images, les costumes, les pratiques devraient ressusciter la République batave et lui conférer une originalité (politique, économique, sociale, culturelle) qu'on lui a souvent déniée.
\end{abstract}

Mots clés : révolution batave ; République batave ; historiographie révolutionnaire.

Les années 1980 ont été décisives pour la recherche historique néerlandaise : elles ont vu la revalorisation du mouvement patriote (1781-1787) et, par contrecoup, la réactualisation de la période suivante, longtemps méconnue et mésestimée parce qu'interprétée comme "période française ". Tout cela est bien connu depuis les articles fondateurs de Willem Frijhoff et de Joost Rosendaal ou ceux de Niek van Sas et de Wayne Te Brake (1). En 1977, le livre de Simon Schama avait ouvert la voie sans pour autant

(1) W. FRIJHOFF et J. ROSENDAAL, «La Révolution régénérée : nouvelles approches et nouvelles images de la révolution néerlandaise ", dans L'image de la Révolution française, (éd. M. Vovelle), Pergamon Press, I, 1990, pp. 543-561. N.C.F. VAN SAS, "Simon Schama : exponent van een nieuwe orthodoxie?", Kleio, XIX, 1978, pp. 276-283. Du même auteur, "De Nederlandse revolutie van de achttiende eeuw", Bijdragen en Mededelingen betreffende de Geschiedenis der Nederlanden (abrégée par la suite, B.M.G.N.),

Annales historiques de la Révolution française - 2001- $N^{\circ} 4$ [1 à 23] 
«révolutionner » l'interprétation, puisqu'il se fondait pour une bonne part suŕ les travaux des trois grands historiens de la révolution batave: Colenbrander, Geyl et surtout sur les conclusions de De Wit, qui, un des premiers, avait insisté sur l'originalité de la révolution néerlandaise et sur les liens qui reliaient 1780-1787 et 1795-1813 (voir 1848, date où s'impose une véritable démocratie moderne, dotée d'une constitution libérale) (2).

La commémoration de la révolution batave n'a pourtant pas eu l'ampleur de celle des patriotes. Alors que 1987 a donné lieu à nombre d'expositions et de colloques, suivis de publications (catalogues, recueils d'actes, numéros spéciaux de revues, thèses universitaires) (3); alors que 1989 a été prétexte à des études comparatives ou à des mises au point sur des sujets propres aux Pays-Bas (codification, patriotisme versus nationalisme, dissidents, protestants et révolution, femmes révolutionnaires et contre-révolutionnaires, unification financière, administration et francisation) (4), la «libération » de janvier 1995 n'a pas fait battre le cœur des foules, ni même celui des historiens et des gouvernants. Peu nombreux ont été les colloques, et les publications, qui peu à peu paraissent, ont tout autant pu être stimulées par les commémorations précédentes (5). Le témoignage le plus éloquent de l'amnésie où demeurait confinée la révolution batave - amnésie collective stimulée par la dynastie d'Orange, peu encline à réactualiser un passé dont elle était absente, en tant que monarchie du moins - réside sans

$\mathrm{n}^{\circ}$ 100, 1985, pp. 636-646 et «The Patriot Revolution : New Perspectives ", dans The Dutch Republic in the Eighteenth Century. Decline, Enlightenment and Revolution, (éd. M.C. Jacob et W.W. Mijnhardt), Cornell University Press, 1992, pp. 91-122. W. TE BRAKE, " How Much in How Little? Dutch Revolution in Comparative Perspective », Tijdschrift voor Sociale Geschiedenis, $n^{\circ} 4$, 1990, pp. 349-363. Du même auteur, "Staking a new claim to an old revolution : A review article », Tijdschrift voor Geschiedenis (abrégé par la suite, T.v.G.), n 104, 1991, pp. 15-23.

(2) S. SCHAMA, Patriots and Liberators : Revolution in the Netherlands 1780-1813, London et New York, 1977. Sur les critiques faites à Schama, voir surtout les articles de N.C.F. VAN SAS, et notamment celui de 1992. Avant De Wit, Pieter Geyl avait corrigé les anachronismes de Colenbrander et rendu à l'époque son caractère "national ", mais niait que le radicalisme batave fût réellement autochtone.

(3) F. GRIJZENHOUT, N.C.F. VAN SAS, W.W. MIJNHARDT (éd.), Voor vaderland en vrijheid. De revolutie van de patriotten, Amsterdam, 1987. H. BOTS et W.W. MIJNHARDT (éd.), De droom van de revolutie, Amsterdam, 1989. Th. VAN DER ZEE, P. THISSEN et J. ROSENDAAL (éd.), 1787. De Nederlandse revolutie?, Amsterdam, 1988. W. BERgSma (éd.), For uwz lân, wyv en bern. De Patriottentijd in Friesland, Leeuwarden, 1987. « Holland 1787 », Holland, regionaal historisch tijdschrift, $n^{\circ} 19,1987$. « O vrijheid !... Aspecten van de patriottenbeweging in stad en gewest Utrecht », Jaarboek Oud-Utrecht 1987, Utrecht, 1987. Ce sont là quelques exemples, il en est d'autres. Voir notamment W. TE BraKE (1991).

(4) Voir note précédente et les numéros spéciaux des revues B.M.G.N. et de T.v.G. de 1989. On peut encore citer W. FRIJHOFF et R. DEKKER, Le voyage révolutionnaire, Hilversum, 1991.

(5) Parmi les colloques de 1994-1995, celui de Louvain, B. VAN HET HERTEN (éd.), Het vuur van de Revolutie, De Achttiende Eeuw, n² 28, 1996; celui de Paris, A. Jourdan et J. TH. LeERSSEN (éd.) Remous révolutionnaires. République batave, ammée française, Amsterdam, 1996; à Utrecht et Nimègue, sur la révolution batave et le droit : O. MOORMAN VAN KAPPEN et E.C. COPPENS (éd.), De Bataafse omwenteling en het recht, Nijmegen, 1997; à Amsterdam, sur l'Institut royal, W.P. GERRITSEN (éd.), Het Koninklijke Instituut en de bevordering van wetenschap en kunst, Amsterdam, 1997; et la même année, à La Haye, une exposition organisée par N.C.F. VAN SAS et F. GRIJZENHOUT, ayant pour titre : Denkbeeldig vaderland, Kunst en politiek in de Bataafs-Franse tijd 1795-1813, La Haye, 1995. 
nul doute dans le fait qu'elle était quasiment passée sous silence dans l'enseignement secondaire, jusqu'en 1997.

Cette période méconnue est d'autant plus passionnante qu'elle présente un intérêt à la fois pour une meilleure appréhension de l'histoire des Pays-Bas; pour l'histoire de la France, si étroitement impliquée dans les vicissitudes des Républiques-sœurs, et, notamment, dans celles de la République batave - surtout du point de vue financier; pour l'histoire des révolutions en général, et donc pour l'histoire comparatiste qui s'interroge sur les influences, les interactions, les transferts, les rejets ou les configurations nationales. De ce point de vue, rendre accessibles à un public étranger les travaux récents des historiens néerlandais doit enrichir l'histoire européenne de la période $1780-1815$.

\section{L'historiographie depuis 1977}

Inutile donc de revenir sur les débats des années 1780 , dont les enjeux ont été fort bien analysés dans les articles de Frijhoff, Rosendaal, van Sas et Te Brake. Inutile non plus de rappeler combien l'historiographie française s'est peu intéressée à rendre leur juste place aux révolutions européennes. $L a$ Grande Nation de Jacques Godechot souligne avant tout l'influence française en Europe et néglige quelque peu les spécificités nationales et les événements d'avant 1789. Albert Soboul minore la portée des révolutions américaine, anglaise, italienne pour majorer celle de la Révolution française. Dans deux ouvrages récents, Michel Vovelle suit en somme l'élan impulsé par Godechot, puisqu'il amplifie le rôle joué par la Grande Nation dans l'éclosion des jacobinismes étrangers. Plus que ses prédécesseurs, Vovelle valorise certes le mouvement révolutionnaire italien, mais minimise l'originalité du modèle batave avant d'insister sur l'impact en Europe de l'exemple français (6). De fait, c'est à un historien américain - Robert Palmer - que l'on doit la première synthèse qui rende pleinement justice à la « révolution atlantique » et c'est lui aussi qui a encouragé les Néerlandais à se pencher sur leur tumultueux passé. Si l'ouvrage de Palmer a pour ainsi dire inspiré De Wit (7), celui de Schama, semble-t-il, est cause de la prolifération des travaux actuels. À peine publié, il a pourtant suscité une avalanche de critiques. Car, si les historiens s'accordent pour louer le style de l'ouvrage, nombreux sont ceux qui regrettent que, malgré l'apport de sources inédites, l'histoire de la

(6) M. Vovelde, Les jacobins. De Robespierre à Chevènement, La Découverte, 1999. M. VoVELLE, Les républiques-sceurs sous le regard de la Grande Nation 1795-1803. De l'Italie aux portes de l'Empire ottoman, limpact du modele républicain français, L'Harmattan, 2000. De fait, il s'agit d'un recueil d'articles qui portent principalement sur l'Italie et quelque peu sur la Suisse.

(7) Entre autres grâce à l'article * Much in little. The Dutch Revolution of 1795 ", Joumal of Modem History, XXVI, 1954, pp. 15-35. 
période n'y soit en rien renouvelée. La phase radicale est jugée non nécessaire, voire inutile; les généralisations sont trop rapides et parfois incorrectes; la continuité entre 1780 et 1848 est trop poussée. On pourrait ajouter que, dans cet ouvrage, Schama fredonne déjà le refrain qui sera celui des Citizens, à savoir que la révolution, synonyme de violence et de sang, était aussi peu nécessaire en France que ne l'était le coup d'État radical en Hollande, du 22 janvier 1798. Voilà qui ne nous renseigne pas vraiment sur les spécificités du «radicalisme » batave (janvier-juin 1798), sur les raisons plus retorses à la source du coup d'État des modérés de juin 1798 et sur le rôle joué à l'arrière-plan par les Français - Talleyrand, notamment. Schama interprète ce second coup d'État comme inéluctable, car, par leurs épurations, répressions et abus, les radicaux se seraient aliénés la population et les élites révolutionnaires. Maarten Prak qui a étudié de très près la révolution dans le Brabant, et, en particulier, à Bois-le-Duc (baptisé Bois-le-Brutus, en 1795) nous signale qu'outre les épurations des assemblées primaires qui ont exclu trente pour cent des électeurs potentiels, aucune mesure répressive n'a touché les modérés ou les opposants (8). Les études de Renger de Bruin sur Utrecht ne sont pas en mesure d'apporter des précisions semblables, mais l'auteur n'en remarque pas moins des décalages entre les votes de 1797 et de 1798 (9), qui ne peuvent tous s'expliquer par les épurations - il n'en remet pas pour autant en cause les interprétations de Schama. Il serait souhaitable cependant de vérifier l'impact réel des épurations, l'ampleur des répressions dans toutes les provinces et d'analyser les choix des assemblées primaires et leurs préoccupations. Ce n'est qu'après une telle enquête que pourront être tirées des conclusions vraiment fiables, non seulement sur le régime né le 22 janvier 1798, mais aussi sur son radicalisme et sur celui des sections, clubs et assemblées.

Sur ce qu'il en est des évolutions historiographiques actuelles, Wayne Te Brake, historien américain dont le livre sur la province d'Overijssel fait référence auprès des spécialistes de la période, constate un net glissement (10). Depuis Schama, en effet, l'histoire politique a délaissé le

(8) M. PRAK, Republikeinse veelheid, democratisch enkelvoud. Sociale verandering in het Revolutietijdvak's-Hertogenbosch 1770-1820, Nijmegen, 1999. La plupart des députés fédéralistes notoires ont certes été incarcérés jusqu'en juin, dans une ancienne résidence du Stadhouder - alors que Delacroix et Ducange voulaient les envoyer à Cayenne.

(9) L'auteur a compté les pourcentages d'opinions négatives de 1797 et les a comparées avec les opinions positives de 1798 , qui s'avèrent divergentes, ce qui implique que les rejets ne se sont pas automatiquement transformés en acceptations et que les votes positifs de 1797 ne se transforment pas automatiquement en 1798 en votes négatifs. De plus, l'épuration n'a pas mené à une acceptation générale. Il y eut des voix contre le projet radical. R. DE BRUIN, Burgers op het kussen. Volkssoevereiniteit en bestuurssamenstelling in de stad Utrecht, 1795-1813, Zutphen, 1986, pp. 171-173.

(10) W. TE BRAKE, Regents and Rebels. The Revolutionary World of an Eighteenth-Century Dutch City, Basil Blackwell, 1989. cf. ses articles : "Burgers en boeren in the Dutch patriot revolution", dans 1987. De Nederlandse revolutie?, Amsterdam, 1988; "How much in how little? Dutch Revolution in comparative perspective ", op. cit. 
« centre » pour s'intéresser à la périphérie; les personnages de premier plan cèdent la place aux acteurs secondaires, voire anonymes; les révolutionnaires n'ont plus seuls droit de cité mais aussi les contre-révolutionnaires (11); et de conclure sur le consensus en vigueur : à savoir que les deux périodes 1781-1787 et 1795-1813 sont liées et qu'elles participent de l'ample processus révolutionnaire si bien perçu par Palmer. Te Brake, luimême spécialiste de l'histoire sociale régionale, interroge le processus de politisation et de mobilisation des élites et du peuple; la diversité politique, économique et sociale des coalitions; au lieu de focaliser sur les intentions, il encourage à prendre en considération les effets et la diversité des violences et des changements, tandis qu'il est convaincu que les révolutions manquées ne sont pas sans conséquences.

Évoquant les travaux de Niek van Sas, de Willem Frijhoff et de Wijnand Mijnhardt, Te Brake note aussi la priorité désormais conférée à la culture politique : qu'il s'agisse des symboles et des rituels, des discours politiques et de leurs mutations; des sociétés littéraires et philanthropiques ou de la presse. Et, il est vrai, que, durant les années quatre-vingts, les historiens néerlandais ont changé de cap : pour adopter les perspectives des historiens anglo-saxons (Baker, Hunt, Jacob), ou adapter au cas néerlandais les approches d'historiens français (Roche, Vovelle, Chartier, Furet, Ozouf). L'histoire-récit a cédé la place à une histoire-problème; les événements sont occultés au profit de thèmes culturels précis, qui restituent une société, ses attentes, ses combats et les enjeux politiques qu'ils recouvrent.

Niek van Sas, responsable pour une part de l'introduction dans le programme scolaire de la période des patriotes et de la République batave, explore les discours et les pratiques qui mènent à la politisation des peuples et interroge les liens qui se nouent entre culture et patriotisme, entre Lumières et Révolution (12). Dans les nouveaux médias : imprimés (journaux, livres, pamphlets, projets de constitution), sociétés (tout d'abord sociétés littéraires, philanthropiques, maçonniques, ensuite, sociétés politiques ou économiques), images et rituels (caricatures, estampes, tableaux, bustes, décors, serments, parades, banquets, pétitions), émerge un discours politique qui traduit (ou se traduit par) des pratiques inédites ou qui en renouvelle de plus anciennes. À ces travaux s'ajoutent ceux de Wijnand Mijnhardt, consacrés aux sociétés culturelles des Pays-Bas. Mijnhardt

(11) Cf les études de W. Velema sur Elie Luzac. Entre autres, Enlightenment and conservatism in the Dutch Republic. The political thought of Elie Luzac (1721-1796), Assen/Maastricht, 1993. Et le numéro spécial de la revue, T.v.G., $\mathrm{n}^{\circ} 102,1989$, consacré à « Révolution et Contre-révolution ».

(12) N. C. F. VAN SAS, “Opiniepers en politieke cultuur » dans Voor Vaderland en Vrijheid. De revolutie van de patriotten, (éd. F. Grijzenhout, W.W. Mijnhardt, N. C. F. VAN Sas), Amsterdam, 1987. Voir aussi "Tweedragt overal : het patriottisme en de uitvinding van de moderne politiek », dans De Droom van de Revolutie. Nieuwe benaderingen van het Patriottisme, (éd. H. Bots et W. W. Mijnhardt), Amsterdam, 1988 ou «La sociabilité politique des patriotes bataves », Annales historiques de la Révolution française, LXI, 1989, pp. 210-224. 
analyse la sociabilité politique et l'esprit critique qui naît au contact de ces institutions locales, publiques ou privées (13) et brosse une image fiable de ce que furent les Lumières néerlandaises. Depuis plus longtemps, Willem Frijhoff a opté pour une approche anthropologique et s'intéresse notamment aux relations entre les peuples, aux représentations (tant nationales qu'internationales). On lui est redevable de plusieurs articles fondateurs (14), qui ont encouragé le comparatisme en histoire et enrichi notre compréhension des cultures - française et néerlandaise. Il est un des premiers à avoir stimulé ici les travaux sur les interactions en histoire (15). Un autre spécialiste de la période, Frans Grijzenhout, a consacré sa thèse aux fêtes des révolutions patriote et batave. Depuis, il a publié nombre d'études sur l'iconographie révolutionnaire et a organisé plusieurs expositions : à la Haye, avec Van Sas où tous deux défendaient l'idée d'une «patrie imaginaire » dans l'art et la politique des années 1795-1813, et, en 2000, pour célébrer le bicentenaire du Rijksmuseum, où Grijzenhout réactualisait «Louis Napoléon et le musée royal » (16). Aux yeux de ces nouveaux historiens, discours, images, écrits et sociétés constituent une culture, une sociabilité politique aux Pays-Bas mais aussi un peu partout en Europe. Et c'est plus précisément dans ce phénomène commun à l'Occident que résiderait la révolution «atlantique » chère à Palmer - et à Godechot. Mais c'est dire aussi que l'invention de la politique moderne ne serait pas l'apanage de la France (17).

À la lumière des recherches nouvelles, il est apparu de plus en plus évident que les Pays-Bas avaient réussi l'exploit de marier Lumières et religion, et, que le transfert de sacralité, à l'œuvre dans la Révolution française, n'avait ici aucune raison d'être. De même, les institutions culturelles existantes étaient aptes à transformer en actes les aspirations plus ou moins conscientes des patriotes des années 1780 (Frijhoff, 1988). Le pluralisme religieux, l'absence de féodalité et la structure fédérée de la République des

(13) W. W. MunhaRdT, Tot Heil van het Menschdom. Culturele genootschappen in Nederland, 1750-1815, Amsterdam, 1987. Voir aussi l'excellent recueil d'articles : The Dutch Republic in the Eighteenth Century. Decline, Enlightenment and Revolution, op. cit.

(14) W. FRIJHOFF, « Hoe revolutionair kan revolutie zijn? Gedachten over een vergelijkende balans van de revolutionaire mentaliteit in Frankrijk en in de Lage Landen », in 1787. De Nederlandse revolutie?, op. cit., pp. 41-51. Voir encore, "Verfransing? Franse taal en Nederlandse cultuur tot in de revolutietijd ", B.M.G.N, $\mathrm{n}^{\circ} 104,1989$ et "Ĺévidence républicaine : les Bataves au passé, au présent et au futur", A.H.R.F., $\mathrm{n}^{\circ}$ 2, 1994, pp. 179-194.

(15) Voir à ce sujet, Remous révolutionnaires. République batave, armée française, op. cit.. Ou la communication au colloque de Fribourg (Suisse), A. JOURDAN, «La République éternelle et le Directoire français. Le poids des représentations dans la révolution batave » (à paraitre) ou « Le(s) discours batave(s) sur la Grande Nation. La cigale et la fourmi », in Du Directoire au Consulat.2. Lintégration des citoyens dans la grande nation, (éd. H. Leuwers), Lille, 2000.

(16) F. GRIJZnhout, Feesten voor het Vaderland. Patriotse en Bataafse feesten 1780-1806, Zwolle, 1989. Avec N. C. F. VAN SAS, Denkbeeldig Vaderland. Kunst en politiek in de Bataafs-Franse tijd 1795-1813, La Haye, 1995 et F. GRIJZENHOUT, Een koninklijk Museum. Lodewijk Napoleon en het Rijksmuseum 18061810, Zwolle/Amsterdam, 1999.

(17) N. C. F. VAN SAS, « The patriot revolution : new perspectives, op. cit., p. 118. 
Provinces-Unies promettaient des changements plus faciles qu'en France, où, pour réformer le système, il importait au préalable de révolutionner la société tout entière - avec les dangers afférents. Le peu de violence qui ponctue les journées patriotes ou révolutionnaires est due à cette structure, à cette configuration atypique (18). S'y ajoutent la sociabilité et l'apprentissage politiques des années 1781-1787. À la sociabilité mondaine et littéraire succède de fait une intense politisation, suscitée par la défaite batave dans la guerre qui l'oppose à l'Angleterre (1781-1784). La défaite ravive le sentiment de déclin, déjà perceptible vers 1750 où avait été lancée une offensive pédagogique et moralisatrice en vue de rendre au peuple batave ses vertus anciennes et à la patrie sa prospérité d'antan. Intéressante dans ce contexte est l'articulation entre progrès et déclin. Les Lumières hollandaises claironnent leur foi dans le progrès, mais entonnent l'antienne de la décadence due notamment à la dépense, au luxe et à la corruption (19). D'où la nécessité d'une éducation et la réactualisation du républicanisme antique, revu et corrigé par les humanistes et les philosophes. Avec le retour du républicanisme, fort compatible du reste avec les valeurs des Lumières, resurgit le patriotisme. Ce patriotisme agressif et extraverti durant la période de la guerre anglo-batave, doit-il pour autant se confondre avec un proto-nationalisme? L'hypothèse, partagée par plusieurs de nos auteurs (20), est problématique. D'une part, elle a tendance à assimiler patriotisme et nationalisme; d'autre part, elle perçoit la «nationalisation de la culture », à l'œuvre depuis 1750 , comme le signe de l'éclosion d'une identité nationale. Le peuple batave serait un tout, Un et Indivisible, ainsi que le veut la théorie des caractères nationaux. Mais cette théorie, née sous l'Antiquité, retrouvée sous la Renaissance et réactualisée sous une forme plus sophistiquée par Montesquieu, est-elle en mesure de convaincre un peuple particulariste qu'il constitue une nation? Ce que semblent prouver les travaux récents sur la révolution batave, à l'inverse, c'est que le peuple eut du mal à se confondre avec la nation, en raison de ses attachements locaux. De là ses réticences à l'uniformisation et à la centralisation. Il est vrai que provinces et localités ne réagissent pas de la même façon. Le grand village de Tilbourg se distingue ainsi de la ville de Bois-le-Duc, alors que tous deux sont situés dans le Brabant. Une fois obtenue l'égalité entre ville et campagne à laquelle aspirait le village, son patriotisme s'affaiblit, tandis que la foi catholique dominante l'emporte haut la main sur tout sentiment national. À l'inverse de

(18) Cf. W. TE BRAKE, "Violence in the Dutch Patriot Revolution ", dans Comparative Studies in Society and History, $\mathrm{n}^{\circ} 30,1988$, pp. 143-163. Demeure la question de savoir dans quelle mésure l'intervention prussienne de 1787 et celle de la France en 1795 ont freiné d'éventuelles dérives.

(19) Discours commun aux élites roturières de l'Europe des Lumières, notamment dans les pays protestants.

(20) N. C. F. VAN SAS, Talen van het Vaderland. Over patriottisme en nationalisme, Amsterdam, 1996. W. W. MIJNHARDT, “ Natievorming in het revolutietijdvak», B.M.G.N., n 104, 1989, pp. 546-553. Sur ce point seulement, nous nous séparons de nos deux éminents historiens. Encore ces remarques ne sont-elles pas définitives. 
Bois-le-Duc, plus diversifié religieusement, où s'affermit peu à peu l'idée d'une identité nationale. Des conclusions définitives ne sauraient donc être établies avant que de nouvelles études de cas ne viennent compléter le tableau et permettre une synthèse véritable.

Du patriotisme «patriote » à celui des Bataves, un glissement se fait jour, stimulé par l'émergence du concept de «nation» (21). La patrie régresse; la nation progresse, mais c'est encore et toujours un concept à usage socio-politique. À la fin des années 1790 , il acquiert certes une dimension comparative : implicite, mais presque superlative, quand Bonaparte invente et salue «la Grande Nation » ou, explicite, mais plutôt neutre, quand Ockerse publie sa Caractérologie générale, où il confronte Hollandais et Français et signale l'identité de caractère des peuples en révolution (22). Entre-temps et au gré des réformes unitaristes, la forme adjective s'est imposée; le sentiment national s'est exacerbé. Mais, si ce sentiment paraît s'être assez répandu en France, dans les années 1800, à cause des guerres et des traumatismes vécus, ne demeure-t-il pas en Hollande l'apanage des élites et des politiques, jusqu'en 1806, voire 1813 (23)? Le nationalisme constaté dans les années 1806-1813 tendrait à le prouver, puisqu'il concerne la haute culture : beaux-arts, poésie, littérature. La même chose vaut du reste en Allemagne, où ce qui préoccupe les élites durant l'épopée impériale a peu de choses à voir avec ce qui inquiète ou réjouit le peuple (24).

De fait, il paraît quelque peu abusif de déceler dans le patriotisme « extraverti » (1781-1787/1799) ou « introverti » (1800-1813) un nationalisme moderne - à moins de l'entendre comme un nationalisme élitaire, visant à réunir les citoyens néerlandais autour des idéaux (successivement) réformateurs, patriotiques, révolutionnaires, unitaristes, nationaux. Dans ce sens, ce serait plutôt l'expression d'une volonté politique, aspirant à transformer un pays en «nation» pour les besoins de sa cause. Le redécoupage des provinces en départements, entamé en 1798 et réalisé entre 1799 et 1802, participe de cette stratégie. Le gouvernement entendait par là anéantir l'esprit fédéraliste, habituer le peuple à penser la patrie sur un mode nouveau

(21) Voir l'article de N. C. F. VAN SAS dans ce numéro. Ou, J. Th. LeERSSEN, Nationaal denken in Europa. Een cultuurhistorische schets, Amsterdam, 1999, pp. 49-52. Notons qu'en France, l'édit de 1717 actualise le terme, quand il évoque «les droits de la nation », J.-M. CARBASSE, Introduction historique au droit, PUF, 1998, p. 269.

(22) W. A. OCKERSE, Ontwerp tot ene algemeene charakterkunde, Amsterdam, 1797. Il s'agit du 3 e volume. pp. 282-295 (identité des caractères) et pp. 134 ; pp. 158-159; pp. 229-234.

(23) Notons aussi, comme le fait J.-C. MARTIN, «les lourdes conséquences » du principe du droit des peuples à disposer d'eux-mêmes, en ce sens qu'il dissocie les nations des États. Contre-révolution, Révolution et Nation en France 1789-1799, Seuil, 1998, p. 129 et p. 130.

(24) Voir à ce sujet, S. WoOlf, Napoléon et la conquête de l'Europe, Paris, 1990 et M. BROERS, Europe under Napoleon, 1799-1815, London, 1996. Ou A. JouRdaN, L'empine de Napoléon, Paris, 2000. Tous trois contestent que, même sous l'Empire, on puisse parler de naissance des nationalismes et des nationalités. Tout au plus d'un sentiment patriotique, qu'on peut à la rigueur appeler «national ". Notons enfin que J. JoOR (cf. note 56) ne décèle pas non plus dans les révoltes de 1806-1813 de traces « nationalistes". 
et faciliter la rentrée des impôts. Qu'importait que les nouveaux départements, baptisés à la mode du jour, ne coïncident pas avec les autres divisions - celles des "waterschappen", qui s'occupaient des eaux, des digues et des polders ou avec les districts judiciaires! L'essentiel était de faire œuvre d'acculturation, de sorte à créer l'Unité et l'Indivisibilité. Le nouveau système eut du mal à se faire accepter. En 1810, Napoléon le réactualisera, tout en $\mathrm{y}$ ajoutant sa touche particulière. L'objectif, entre-temps, avait changé (25).

Il faudra que s'installe et s'affirme auprès de citoyens récalcitrants l'État unitaire (26), contraint de compenser l'impersonnalisation qui lui est propre par une valorisation du sentiment national - pour attacher à l'ÉtatNation des sujets par trop individuels ou particularistes, et ce, par le biais de l'école, de l'armée, de la politique, voire d'une infrastructure routière avant que naisse le nationalisme moderne (de masse). Jusque-là, mieux vaut parler de patriotisme. Or, patriotes, les Néerlandais n'avaient cessé de l'être depuis leur légendaire résistance contre le Goliath espagnol. Le XVIII' siècle leur proposa une identité culturelle (27), où ils figuraient en dignes républicains, dans le même temps où on leur demandait de ressembler à cette image. La République batave ira plus loin et, à l'instar de la France de la Révolution, envisagera de régénérer la nation. Ce sera en vain. Les Bataves n'étaient plus ceux de Tacite; ils tenaient à leur identité locale ou provinciale et percevaient la nationalisation comme synonyme de nouvelles contraintes.

\section{Nouveaux problèmes, nouvelles approches, nouveaux objets}

Que le nationalisme moderne ne se soit pas emparé des esprits bataves dans les années 1780-1813, c'est ce dont témoignent en somme plusieurs études locales portant sur des provinces ou des villes spécifiques. Celle de Tom Nieuwenhuis qui traite des patriotes et des orangistes dans trois

(25) J. ROSENDAAL, « Diviser pour régner. Le système départemental comme stratégie d'unification aux Pays-Bas (1780-1801)", dans Le Département : hier, aujourd'hui demain. De la Province d̀ la Région, de la centralisation à la décentralisation, (éd. G. Chianéa, R. Chagny, J.-W. Dereymez), Grenoble, 1994, pp. 431-440.

(26) N'oublions pas de surcroît qu'il y eut une période (entre 1750 et 1799) où les Lumières et leurs héritiers, conscients de leur immersion dans le temps et dans l'espace, songeaient non seulement à œuvrer en faveur du progrès de l'esprit humain et de la civilisation, mais aussi et encore à créer une république universelle, synonyme de paix perpétuelle. Cf. A. Jourdan, «Napoléon et la paix universelle. Utopie et réalité ", colloque Napoléon et l'Europe (La Roche sur Yon, juin 2001), actes à paraître.

(27) Pour W. Frijhoff, l'idée d'une identité culturelle commune aux Pays-Bas aurait été la condition de l'acceptation de l'unitarisme. Et non l'inverse. "Het Zelfbeeld van de Nederlander in de achttiende eeuw. Een inleiding ", Achttiende Eeuw, op. cit, pp. 5-28. Le problème ici aussi, c'est que ces auto-représentations sont l'apanage des élites qui pensent, gouvernent et écrivent, et non celle du peuple dans son ensemble. Il est vrai que la culture était diffusée en néerlandais depuis 1750 environ, par le biais de la presse spectatoriale et des sociétés, ce qui a pu avoir quelque influence sur les classes moyennes, mais cela reste à prouver. 
villages de Hollande confirme la tendance générale à l'autonomie. Soumis et dépendants des villes qui exercent sur eux une autorité quasi illimitée, les villages aspirent à l'indépendance. Leur choix entre 1780 et 1787 est fortement motivé par des intérêts locaux spécifiques. Selon les dangers qu'ils pressentent, ils optent pour le parti patriote ou pour le stadhouder. Ransdorp et Holisloot, qui craignent la suprématie croissante de Durgerdam, village voisin patriote, sont pour une grande part orangistes. Bathmen, village de l'Overijssel, menacé par des investisseurs de la ville, prend parti pour les patriotes, sans soupçonner qu'au nom de l'égalité, ces derniers risquent eux aussi de porter tort à la communauté. Les clivages socio-économiques engendrent donc bien souvent des clivages politiques, qui expliquent que, malgré leur particularisme, certains villages se sont accommodés de la Constitution de 1798, parce qu'elle les libérait de l'emprise de la ville (28) - et non pour entrer dans la ronde des nations.

$\mathrm{Si}$ aucune synthèse n'est venue renouveler l'ouvrage de Schama, les travaux parus ces dernières années sur les villes et provinces néerlandaises sont fort précieux, en ce qu'ils nous renseignent sur les rapports de force, les coalitions, les mobilisations et les manifestations, mais aussi sur les divisions et les résistances à la centralisation et à l'unification. W. Te Brake et M. Prak mettent ainsi en avant les conflits qui déchirent les patriotes de la première heure, dès que sont prises des mesures qui menacent l'existence ou la primauté des corporations. La ville d'Ancien Régime et la structure complexe qui la caractérise sont ici ressuscitées. Les alliances séculaires entre élites et classes moyennes se dénouent dès que sont menacés les intérêts particuliers. Car, si la tradition veut que les divers groupes sociaux s'allient contre toute menace venue de l'extérieur (le stadhouder, en principe), l'alliance se brise quand, cette menace disparue, les classes moyennes revendiquent une participation politique que les élites répugnent à leur accorder. De même, patriotes ou non, celles-là exigent encore et toujours une autonomie municipale, une citoyenneté locale, un rôle de premier plan pour les milices bourgeoises. Or, en 1795 , et contrairement à 1787 , il ne s'agit plus de lutter en faveur des libertés locales et de restaurer une constitution violée par un apprenti-tyran, mais, à l'inverse, d'accomplir et d'achever ce dont n'avait jamais osé rêver le prince d'Orange, à savoir l'unité et la centralisation. Cette nouvelle tendance menaçait non seulement les classes moyennes et les corporations, mais aussi les élites provinciales et municipales, qui eurent bien du mal à se soumettre aux lois et directives en provenance de La Haye. Prak observe ainsi jusqu'en 1806 plusieurs résistances et heurts entre autorités locales, provinciales et nationales, tandis que les

(28) T. NIEUwenhuis, Keeshonden en Prinsmannen. Durgerdam, Ransdorp en Holisloot (1780-1813), thèse polycopiée, 1986. W. TE BRAKE, «Revolution and the Rural Community in the Eastern Netherlands ", Class Conflict and Collective Action, Sage Publications, 1981, pp. 53-72. 
corporations parviennent bon gré mal gré à subsister jusqu'en 1808 - alors que la loi les supprime dès 1798 .

Là où Te Brake explore les modalités de politisation dans trois villes de l'Overijssel et décèle de grandes divergences; là où Prak souligne l'importance des corporations pour les classes urbaines et note le conflit qui surgit entre république plurielle et démocratie unitaire, Renger de Bruin interroge le processus de démocratisation et de politisation dans la ville d'Utrecht à la lumière des élections de 1795 à 1813 . L'analyse au niveau municipal permet à l'auteur de constater la réalisation effective des idéaux révolutionnaires. Par le biais des élections, les citoyens bataves firent leur entrée en politique et participèrent directement à l'administration de leur cité. La démocratisation est perceptible surtout au niveau local où le peuple élit le conseil municipal, les membres des commissions et plusieurs fonctionnaires et membres du tribunal. Comme à Bois-le-Duc, où la municipalité dut affronter les velléités démocratiques et corporatistes de la Société patriotique du cru, Utrecht fut confronté aux résistances et initiatives des assemblées de quartier et des assemblées primaires, dont le pouvoir, il est vrai, était limité du point de vue provincial et national, mais qui, dans le cadre de l'administration municipale, étaient invitées non seulement à élire des candidats, mais aussi à voter sur des projets fiscaux, projets de marchés, de corporations et à s'exprimer sur les problèmes d'orphelinat ou la législation du mariage. Les assemblées populaires outrepassaient bien souvent leurs tâches, accablant le Conseil municipal de revendications, de pétitions et le freinant dans ses travaux (29).

Le coup d'État des radicaux du 22 janvier 1798 mit fin à cette expérience inédite en ce qu'il confisqua une grande partie des prérogatives locales. L'ironie du sort veut donc que ce soient les radicaux, et non les modérés, qui aient anéanti la démocratie directe. Vu sous cet angle, il y a loin des Jacobins français aux radicaux bataves. Les uns prônent l'égalité et la démocratie directe (30); les autres visent avant tout l'unité et l'indivisibilité, ce qui les incite à museler les voix discordantes - du peuple, des corporations, des municipalités, qui répugnent à céder une parcelle de leur souveraineté (31). Il faudra paradoxalement attendre les réformes de 1801-1803 avant que soient restitués au peuple ses droits. Droits qui ne tardent pas à être à nouveau bafoués par la constitution centralisatrice et

(29) R. DE BRUIN, Burgers op kussen, op. cit., pp. 175-182.

(30) Et même s'il importe de nuancer, car, on le sait, la voie suivie par les Jacobins est plus complexe. C'est la république jacobine qui, à partir du 14 frimaire an II, instaure un État fort et freine les initiatives des sections populaires et de la Commune, au nom ici aussi de l'unité et de l'indivisibilité.

(31) Ils en viennent même à vouloir museler la presse d'opposition, alors que, quelques mois auparavant, ils s'étaient faits les fervents défenseurs de la liberté en ce domaine et que le républicanisme des Lumières ne se conçoit sans elle. Ils se rapprochent donc ici encore de la République de l'an II. W. R. E. VELEMA, « Het debat over de vrijheid van drukpers in de Bataafse Tijd ", in Grondwetgeving 17951806, Haarlem, 1997, pp. 65-81. 
autocratique de 1805 . C'est dire encore que la démocratie directe est synonyme de décentralisation et d'autonomie locale et qu'elle est vouée à être détruite par la nécessité ressentie d'établir une République Une et Indivisible. La modernisation du système financier et fiscal, jugée indispensable pour enrayer la crise, implique en effet une transformation de l'ancienne fédération en un État unitaire, une hiérarchisation et une centralisation du pouvoir politique, une bureaucratisation des services publics, une uniformisation du système fiscal et une augmentation de la pression fiscale - mais mieux répartie (32).

Les études de Renger de Bruin sur la municipalité d'Utrecht, tout comme celles de Prak ou de Te Brake démontrent également que la polarité établie par De Wit entre démocrates et aristocrates est loin de recouvrir une réalité plus complexe. Les Bataves sont divisés non point en fonction d'une lutte des classes avant-la-lettre, mais en fonction d'un large éventail d'intérêts ou de priorités. De ce point de vue, les Français de séjour dans le pays sont plus près de la vérité, quand ils signalent la multitude de partis qui s'affrontent et la difficulté à bien saisir " les nuances dans un pays où par suite des institutions et des habitudes fédératives, chaque province, chaque canton, chaque ville, chaque classe et dans les classes chaque parti a une opinion différente " (33). Ces affrontements ne se font pas seulement en vue de défendre la patrie et la prospérité nationale, mais encore et surtout en vue de protéger des intérêts particuliers - individuels, locaux ou provinciaux - plus ou moins compatibles avec la démocratie (34). À étudier la période franco-batave par le biais des élections, des conclusions s'imposent toutefois qui affinent les travaux antérieurs : tout d'abord sur la composition et la pérennité du personnel politique. Le Conseil municipal de la ville d'Utrecht, élu en mars 1798 se perpétue durant cinq ans malgré sa composition "populaire ", puisqu'il comprend un régent et sept membres des classes moyennes. À Bois-le-Duc, le coup d'État des modérés ne chasse pas tous les radicaux qui siègent à la municipalité. Six demeurent, mais ils ne sont pas à proprement parler issus de classes populaires (35). La révolution batave aurait donc ici et là stimulé la mobilité sociale et les pratiques démocratiques par le biais des élections, du moins jusqu'en 1805. Tandis qu'à partir de 1804 se laisse percevoir un désenchantement - une dépolitisation - qui se traduit par un taux croissant d'abstentions.

(32) Voir l'article de T. PFEIL dans ce numéro.

(33) Lettre de Sémonville à Talleyrand du 11 prairial an IX (A.E., Hollande, vol. 605). Il y aurait ainsi des radicaux ou des modérés fédéralistes, des radicaux ou des modérés unitaristes, des fédéralistes anarchistes, des orangistes purs, des orangistes constitutionnels, sans oublier les aristocrates, etc.

(34) À plusieurs reprises, Gogel et d'autres patriotes signalent ainsi le non-patriotisme des «capitalistes " néerlandais, qui auraient pour quatre-vingts millions de livres sterling en fonds anglais, nonobstant la guerre (A.N., AF IV-1820, pièce 73).

(35) M. PRAK, op. cit., pp. 250-252. 
Les exemples qui précèdent témoignent à la fois des affinités et des décalages entre la situation française et la situation néerlandaise. Bien que les deux pays se retrouvent pour ce qui est des décalages entre discours et réalités; pour ce qui est des résistances aux ingérences de l'État dans les affaires locales (36), la dynamique révolutionnaire diverge fortement, comme divergent la dimension des groupes impliqués et l'ampleur des résistances - toutes deux limitées en Batavie, et même si l'on ne sait toujours pas à combien s'élevait précisément l'opposition orangiste (37). Si les divisions sont légion, elles sont moins féroces qu'en France, car, ici, non seulement, les structures politiques permettent de discuter des problèmes au niveau local ou provincial, mais les hommes sont aussi habitués à se concerter, jusqu'à ce que soit trouvé un compromis. Dans ce pays où les dissidents ont tous leurs institutions sociales et religieuses, où les pouvoirs sont indéfiniment partagés et où la notion de centre est quasiment inconnue, les citoyens peuvent en appeler à trois juridictions : locale, provinciale, «nationale ». La fiscalité est l'affaire des provinces et (pour ce qui est des impôts indirects) des autorités locales, qui veillent sur leurs intérêts. Les provinces littorales endettées - notamment la Hollande - luttèrent ainsi en faveur de l'unification, afin que soit mis en ouvre un système fiscal égalitaire, auquel s'opposaient à l'inverse les provinces de terre, avantagées par l'ancien système.

De fait, nombreux s'accordaient pour accepter la souveraineté populaire, la démocratie représentative, la liberté, la fraternité, voire l'égalité. Et c'est sur l'amalgame des dettes, l'abolition des corporations, la modalité des réformes, voire sur la formulation des principes que s'élevaient les conflits. La révolution batave s'avère avant tout politique et économique, d'autant plus que la République mère ne cessa de pressurer son alliée. Il s'agissait donc de réformer l'État de sorte à ce qu'il redevienne prospère et puissant - tout autant que sous le Siècle d'Or, référence privilégiée -, les hommes de la fin du XVIIIe siècle crurent remédier à la crise par la révolution et l'unification, en vue d'une refonte de l'administration politique et financière (38) et dans l'espoir qu'advienne l'heure de la régénération morale.

Sur le contexte financier et fiscal, deux études font désormais référence. Wantje Fritschy a réactualisé les idées et les discussions sur le problème et noté le rôle clé des années 1795-1801. Tom Pfeil poursuit l'enquête jusqu'en 1810 et examine de très près les mesures mises en œuvre pour assainir les finances néerlandaises. Car, on le sait et on l'a dit, loin d'être le vaste grenier

(36) Sur la France, J.-P. JEsSEnNE, Pouvoir au village et Révolution. Anois 1760-1848, Lille, 1987. R. DUPUY (éd.), Pouvoir local et Révolution. La frontière intérieure, Rennes, 1995.

(37) W. Te Brake donne trois provinces : la Hollande, l'Overijssel et la province de Groningue pour patriotes; la Frise et la province d'Utrecht seraient divisées; la Zélande et le Gelderland seraient orangistes. T. Nieuwenhuis observe que cela diffère selon les villages, mais des chiffres précis font défaut.

(38) À ce sujet, J. DE VRIES et A. V. D. WOUDE, Nederland 1500-1813. De eerste ronde van modeme economische groei, Amsterdam, 1995. Sur les priorités qui s'imposent, voir l'article de N. C. F. VAN SAS dans ce numéro. 
chargé d'or dont rêvaient les Français, les Pays-Bas connaissaient une crise économique depuis le milieu du siècle qui ne fit que s'amplifier au rythme des guerres et des révolutions (39). La dette publique ne cessa de croître et atteignait plus de 1232 millions de florins en 1810, si bien que les impôts augmentèrent à un rythme inouï, jusqu'à accaparer plus de la moitié des revenus des contribuables et un vingtième de leurs propriétés foncières.

L'aspiration à l'uniformisation se fait également sentir dans le domaine du droit et de la codification. La révolution a incité en effet à rassembler et à simplifier ce qui autrefois était épars et hétéroclite. Mais alors que les partis bataves s'affrontaient sur les modalités d'une constitution unitaire, le consensus se fit sur la nécessité de systématiser le droit civil, pénal et judiciaire dans des codes accessibles à tous. Avant même la révolution de 1795, Van de Spiegel, Grand Pensionnaire et fervent orangiste, songeait à un code national. Des concours avaient été ouverts dans plusieurs sociétés éclairées; les patriotes eux aussi avaient poursuivi le rêve d'une codification uniforme, tout comme les réfugiés qui conçurent en 1793 un projet de constitution, incluant une codification du droit. À l'exemple de la France, la République batave ne parvint pas malgré tout à achever cette codification et c'est à Napoléon (et à Louis) qu'est due l'introduction dans le royaume de Hollande d'un code civil et d'un code pénal. Mais le problème et les aspirations étaient donc plus anciennes; elles touchaient la plupart des pays européens, à l'exception de l'Angleterre, satisfaite de son common law (40). C'est dire que la tendance à la classification, à la formalisation et à la systématisation signalée par Foucault déborde largement les sciences humaines. Elle investit jusqu'au domaine juridique et ambitionne une efficacité accrue, en vue du progrès et du bonheur de l'humanité.

Parallèlement, des objets nouveaux attirent l'attention des jeunes chercheurs néerlandais. La "nouvelle génération" - ainsi baptisée par W. Te Brake - a fait école. Les travaux sur la presse, les sociétés et l'imprimé se sont multipliés; la littérature de l'époque est sortie de l'oubli de même que les publications populaires ou les livres pour la jeunesse (41); il y a eu des

(39) W. FRITSCHY, De Patriotten en de financiën van de Bataafse Republiek. Hollands krediet en de smalle marges van een nieuw beleid, 1795-1810, Amsterdam, 1988. T. PFEL, Tot redding van het vaderland. Het primaat van de Nederlandse overheidsfinanciën in de Bataafs-Franse Tijd, 1795-1810, Amsterdam, 1998.

(40) A. H. HUUSSEN Jr., "De betekenis van codificatiegedachte en - praktijk voor de natievorming », B.M.G.N., 104, 1989. En Prusse, le roi avait ordonné dès 1746 la réalisation d'un code des lois; il fut introduit en 1794 seulement. En Autriche, les empereurs avaient introduits un code pénal. En 1811, seulement ce fut le tour du code civil. Voir aussi, J.P. LoOF (éd.), Twee honderd jaar rechten van de mens in Nederland, La Haye, 1994.

(41) À la suite des recherches novatrices de P. J. BuINSTERS ( «De patriot als schoolmeester : Patriotse ideologie in achttiende-eeuwse kinderboeken", in 1787. De Nederlandse revolutie?, op. cit., pp. 100-111), on peut nommer J. LENDERS, De burger en de volksschool. Culturele en mentale achtergronden van een ondenwijshervorming. Nederland 1780-1850, Nijmegen, 1988. J. J. KLOEK, Een begrensd vaderland: de roman rond 1800 : tussen nationaal karakter en internationale markt, Amsterdam, 1997. G. J. JOHANNES, De lof der aalbessen. Over (Noord-)Nederlandse literatuurtheorie, literatuur en de consequenties van kleinschaligheid, 1770-1830, La Haye, 1997. 
recherches synthétiques sur le journal néerlandais, en tant que baromètre du goût (42); des études détaillées sur les sociétés littéraires ou sur le monde de l'édition, telles celles d'Ariane Baggerman qui a ressuscité un univers : celui de la firme A. Blussé et fils, éditeur de Dordrecht. À l'instar de Dorothée Sturkenboom, à qui l'on doit un bel ouvrage sur la sensibilité féminine et les représentations qu'en renvoie la presse spectatoriale au cours du XVIII siècle, Baggerman brosse un tableau des mentalités telles qu'elles transparaissent dans les ouvrages publiés par Blussé, en particulier, dans les manuels pédagogiques qui s'adressent aux parents et éducateurs. L'histoire de l'édition s'y marie avec une histoire culturelle et familiale (43). De la sensibilité féminine aux mentalités familiales, on comprendra que se soient aussi multipliées les études sur l'histoire de la femme à l'époque des Lumières et des révolutions. À la suite de Sturkenboom et de Dekker, Myriam Everard en est une des spécialistes et se passionne notamment pour l'homosexualité féminine au XVIII' siècle et la politisation des femmes sous la Révolution (44). Dans la synthèse à plusieurs mains consacrée à la naissance de la nation néerlandaise (45), Wantje Fritschy confère lui aussi une attention soutenue au problème féminin, tandis que la collection egodocumenten (Verloren, Hilversum), dirigée par Rudolf Dekker et Gerd Jan Johannes, publie des journaux et des correspondances de l'époque, parmi lesquels plusieurs sont écrits par des femmes (46). Les exclus de la société nouvelle ne sont pas seulement les femmes mais aussi les pauvres (peu étudiés jusqu'à présent pour la période qui nous intéresse) et les races dites inférieures. À ce sujet, Angelie Sens réactualise les idées de l'époque sur l'esclavage, les Noirs et l'abolitionnisme. Elle s'interroge en particulier sur «l'énigme néerlandaise » et sur la question de savoir pourquoi, à l'inverse de leurs alliés français, les révolutionnaires bataves n'ont su se résoudre à abolir l'esclavage. C'est dire qu'il s'agit d'une étude où s'entremêlent dimension culturelle et dimension économique, car, si l'abolition s'avérait inconcevable, c'est bien parce qu'étaient en jeu maints intérêts perçus comme

(42) G. J. JOHANNES, De barometer van de smaak. Tijdschriften in Nederland 1770-1830, La Haye, 1995.

(43) D. STURKENBOOM, Spectators van Hartstocht. Sekse en emotionele cultuur in de achttiende eeuw, Hilversum, 1998. A. BAGGERMAN, Een lot uit de loterij. Familiebelangen en uitgeverspolitiek in de Dordtse firma A. Blussé et zoon, 1745-1823, Dordrecht, 2000. Et un ouvrage récemment paru sur les sociétés littéraires, M. DE VRIES, Beschaven! Letterkundige genootschappen in Nederland (1750-1800), Nijmegen, 2001.

(44) M. EVERARD, Ziel en Zinnen. Over liefde en lust tussen vrouwen in de tweede helft van de achttiende eeuw, Groningen, 1994. Citons encore la thèse de Theo van der Meer sur l'homosexualité masculine, considérée comme un crime capital jusqu'à l'introduction du Code pénal (1811): Sodoms zaad in Nederland : het ontstaan van de homosexualiteit in de vroegmoderne tijd, Nijmegen, 1995.

(45) W. FRITSCHY et J. TOEBES (éd.), Het ontstaan van het modeme Nederland. Staats - en natievorming tussen 1780 en 1830, Nijmegen, s.d. (1997), pp. 187-214.

(46) Citons les plus importants : Het Dagboek van Magdalena van Schinne (1786-1805), Hilversum, 1990. Écrit en français, l'ouvrage a été publié aux éditions côté-femmes en 1994 sous le titre Joumal de Magdalena van Schinne; et Clara Cornelia VAN EIJCK, Mijn waarde vrindin. Een Gents joumaal (1790. 1791), Hilversum, 2000 (introduction de J. Rosendaal). 
cruciaux, mais aussi parce que les préjugés relatifs aux Noirs demeuraient vivaces (47).

Parmi les objets encore méconnus, l'histoire religieuse n'est pas des moindres. Or, dans la République des Provinces-Unies, vivotent de nombreuses sectes. Les divisions ici aussi sont nombreuses. Les réformés y sont privilégiés en tant que membres de la religion officielle et, seuls habilités à remplir les fonctions publiques, alors que les dissidents, les catholiques et les juifs ont plus de mal à gravir l'échelle sociale. Arminiens, mennonites, luthériens ont donc accueilli avec enthousiasme la révolution des patriotes. Comme les catholiques et les juifs, ils espéraient des réformes justes et égalitaires. Et, ils furent nombreux en 1795 à entrer en révolution ou à en partager les idéaux (48). Il en va différemment des francs-maçons, dont les opinions politiques étaient partagées et qui ne se retrouvaient pas tous dans les bouleversements révolutionnaires. Contrairement, pourtant, aux pays européens où la franc-maçonnerie fut poursuivie et condamnée au silence, aux Pays-Bas, elle parvient à se maintenir, nonobstant - ou plutôt grâce à ses ambiguités politiques et sociales. Sur ces problèmes, Anton van de Sande et Joost Rosendaal ont publié plusieurs travaux (49), tandis que Simon Groenveld a étudié les clivages religieux et que Simon Vuyk a éclairé la part prise par les Arminiens dans la révolution. La religion, on l'a dit, était essentielle aux Pays-Bas, si bien que, dans les débuts, l'invasion française de 1795 provoqua l'effroi de bien des croyants, qui craignaient une déchristianisation brutale. La France sut pourtant respecter en ce domaine les coutumes, et, personne ne s'étonna de ce que des pasteurs (réformés ou arminiens) et des curés (catholiques) se retrouvent à la tribune de l'Assemblée nationale, parfois dans les rangs des radicaux (50).

Inspirés par les travaux de Frans Grijzenhout, des chercheurs ont réactualisé l'histoire de l'art, et, plus particulièrement celle de la naissance du musée national. Le célèbre Rijksmuseum d'Amsterdam s'avère ainsi être une création révolutionnaire, menée à bien par Louis Bonaparte - qui ne

(47) A. SENS, De neger, slavemij, abolitionisme en het Nederlandse «enigma». Een vergelijkend onderzoek naar de opvattingen over slavemij in Frankrijk en de Republiek (1750-1800), mémoire de DEA, Université d'Utrecht, 1992. Vient tout juste de paraître, A. SENS, Mensaap, heiden, slaaf. Nederlandse visies op de wereld rond 1800, La Haye, 2001.

(48) Encore importe-t-il de nuancer. Les catholiques et les dissidents ne furent pas tous patriotes, tandis que parmi les protestants réformés, il y en a eu beaucoup qui partageaient les idéaux patriotiques. Voir J. VAN OSS et J. ROSENDAAL, «1787: de Nederlandse Revolutie. Een balans", in 1787. De Nederlandse Revolutie?,op. cit., p. 251.

(49) A. VAN DE SANDE, Vrijmetselarij in de Lage Landen. Een mysterieuze broederschap zonder geheimen, Zutphen, 1995. A. V. D. SANDE et J. ROSENDAAL, "Een stille leerschool van deugd en goede zeden". Vrijmetselarij in Nederland in de XVII' en XIX' eeuw, Hilversum, 1995. S. Groenveld, Huisgenoten des geloofs. Was de samenleving in de Republiek der Verenigde Nederlanden verzuild?, Hilversum, 1995.

(50) S. VUYK, De verdraagzame gemeente van vrije christenen. Remonstranten op de bres voor de Bataafse Republiek, Amsterdam, 1995. Voir aussi les articles de J. V. D. BERG et de A. VAN DE SANDE in 1787. De Nederlandse revolutie?, op. cit. ou W. H. DER OUDEN, Kerk onder patriottenbewind. Kerkelijke financiën en de Bataafse Republiek, 1795-1801, Groningen, 1994. 
voulait pas faire moins que son illustre frère. Les saisies d'œuvres d'art du stadhouder et leur séjour au Louvre ont fait l'objet de recherches de doctorat; depuis 1982, Eveline Koolhaas-Grosfeld se passionne pour la politique artistique des années 1780-1840. L'une des premières, elle a signalé la «nationalisation» du goût artistique néerlandais et, entre autres, l'intérêt nouveau pour les paysages bataves. Ses études sur les costumes néerlandais et leurs représentations poursuivent cette enquête (51). D'autres auteurs ont consacré des travaux aux images néerlandaises de la Révolution française et aux œuvres françaises importées aux Pays-Bas. Dans ce contexte et dans le cadre d'une vaste recherche comparatiste, une équipe internationale travaille sur les différentes versions des Tableaux historiques de la Révolution française (52). Les versions hollandaise et allemande sont particulièrement significatives des transferts culturels qui s'opéraient à l'époque et des réceptions ou adaptations diversifiées, auxquelles ils ont donné lieu. Sous cette perspective est également passionnante l'étude des caricatures anti-napoléoniennes, réalisées en Hollande. Contrairement à ce qui est parfois affirmé, les Bataves ont un riche réservoir iconographique d'estampes et de caricatures, ce à quoi l'on pouvait logiquement s'attendre, puisqu'ils connaissent une tradition des plus anciennes dont s'est largement inspirée l'Europe tout entière, et notamment Hogarth (53). De l'époque des patriotes à la chute de Napoléon, les images se sont multipliées pour illustrer les luttes entre Néerlandais et les événements français ou bataves; pour célébrer les héros anciens ou contemporains, et, pour défigurer et disqualifier le "tyran corse". L'ensemble n'est pas encore bien connu et une monographie sur l'art et la gravure révolutionnaires (et/ou contre-révolutionnaires) serait la bienvenue. Jusqu'à présent, ont surtout été réactualisés les décors des fêtes patriotes et révolutionnaires; ou les œuvres classiques (du Siècle d'Or) devant lesquelles se recueillaient des Bataves opprimés par l'impérialisme napoléonien. De même, rares encore sont les études sur l'imaginaire monu-

(51) E. BergVelt, Pantheon der Gouden Eeuw. Van nationale konst-gallerij tot Rijksmuseum van schilderijen, 1798-1896, Zwolle, 1998. F. GRIJZENHOUT, «La patrie réinventée. L'art hollandais dans la période française " in Remous révolutionnaires. République batave, Année française, op. cit., pp. 138-158. E. KOOLHAAS-GROSFELD, « Nationale versus goede smaak. Bevordering van nationale kunst in Nederland 1780-1840», T.v.G., n 95, 1982, pp. 605-636. A. E. Pronk, Les tableaux hollandais au Louvre. Politique artistique et révolution 1794-1815, mémoire de maîtrise, Université d'Amsterdam, 1999.

(52) Les premiers résultats viennent d'être publiés. Bildgedächtnis eines welthistorischen Ereignisses. Die Tableaux historiques de la Révolution française, (éd. Ch. DANELzIK-BRúGGEMANN et R. REICHARDT), Göttingen, 2001. Sur la version néerlandaise, A. JOURDAN, «Die "Tafereelen van de Staatsomwenteling in Frankrijk" oder Die Französische Revolution in den Niederlanden », pp. 85-142. En 2002, le Musée de la Révolution de Vizille consacrera une exposition à ces diverses versions, accompagnée d'un catalogue avec des articles de fond, en français, de Rolf Reichardt, Claudette Hould, Philippe Bordes, Werner Szambien, Annie Jourdan.

(53) E. O. G. HaITSMa MULIER et A. LEYTEN (éd.), Het Nederlandse beeld van de Franse Revolutie, Amsterdam, 1989. Sur les caricatures, H. BAHIA, L’image négative de Napoléon Bonaparte aux Pays-Bas à travers l'exemple de la caricature hollandaise et anglaise, mémoire de maitrise sous la direction de W. Frijhoff, A. Jourdan et G. Deregnaucourt, Lille III, 1999. Sur Hogarth, The Dumb Show. Image and Society in the works of William Hogarth, (éd. F. OGÉE), Oxford, 1997. 
mental des Bataves et sur le réaménagement urbain qui a succédé ou non à la révolution (54). Enfin, les transferts culturels demandent à être approfondis, du point de vue des images, des écrits et des hommes. Tout comme les émigrés bataves auxquels Joost Rosendaal consacre sa thèse, des intermédaires, tel H. J. Jansen, hollandais qui fut polygraphe et traducteur, imprimeur et libraire à Paris, mériteraient d'être mieux connus.

La révolution des patriotes a eu le vent en poupe; la révolution batave et la période qui s'ensuit font actuellement l'objet de divers travaux, entre autres dans le cadre d'un programme de l'Organisation néerlandaise pour la Recherche scientifique (NWO), consacré à « la culture néerlandaise dans le contexte européen. Année charnière $1800 »(55)$. Par contre, les années 1806-1813 font figure de parents pauvres. Les travaux sur le royaume de Lodewijk Napoleon (alias Louis Bonaparte) et sur l'incorporation à l'Empire de 1810 sont l'exception qui confirme la règle. Encore sont-ils loin d'être actuels ou novateurs. La période est impopulaire, tout comme celui sous le sceau duquel elle est marquée. Un seul travail mérite ici d'être nommé : l'opus de Johan Joor qui ouvre et recouvre un chantier passionnant, celui de la réception aux Pays-Bas des nouveautés introduites par Napoléon et des révoltes et protestations populaires qu'elles provoquent (56). Cet ouvrage «révolutionne » en somme l'historiographie traditionnelle, en ce qu'il nous prouve le contraire de ce que l'on nous a affirmé des décennies durant : à savoir que le peuple néerlandais n'est pas l'être apathique, indifférent, calme et ordonné de la fable. Prolongeant pour ainsi dire les recherches de Rudolf Dekker sur les soulèvements populaires en Hollande au cours des XVIIe et XVIIIe siècles, Joor présente un tableau nuancé et précis des actions et réactions des Néerlandais face à la dictature impériale. Cette approche incite à la fois à entreprendre des études de fond sur la mise en place des institutions et sur les actions des hommes (fonctionnaires, habitants, soldats, déserteurs, etc.) des années 1806-1813. Souhaitable serait aussi une étude qui fasse pendant à celle de Silvia Marzagalli sur le négoce maritime et le blocus continental (57); de même, on

(54) Une exception : T. H. VON DER DUNK, «De Bataafse Omwenteling en de bouwkunst. De betekenis van het jaar 1795 voor de Nederlandse architektuur. Een eerste balans ", B.M.G.N., ${ }^{\circ} 111,1996$, pp. 313-343.

(55) Ces travaux ont été nommés plus haut, dans le cadre des études sur les sociétés littéraires, la presse, l'imprimé en général. Les directeurs du programme viennent de publier: J. J. KLOEK et W.W. MIJNHARDT, 1800. Blauwdrukken voor een samenleving. Nederlandse cultuur in Europese context, La Haye, 2001.

(56) J. JoOR, De Adelaar en het Lam. Onrust, Opruüng en onwilligheid in Nederland ten tijde van het Koninkrijk Holland en de Inlijving bij het Franse Keizerrijk (1806-1813), Amsterdam, 2000. On peut encore citer un mémoire de maîtrise sur l'armée royale, M. v.d. HoEven, Van de Weser tot de Weichsel. Het leger van het Koninkrijk Holland en de Duitse veldtochten van Napoleon, 1806., 1807 en 1809, Amsterdam, 1994. Et E.C.G. BouWERS, Het nationale Gerechtshof 1802-1811, Amsterdam, 1997; A. M. ElıAs, Het nationaal Sijndicaat, 1802-1805, Bussum, 1975. 2001.

(57) S. MARZAGAll, Les boulevards de la fraude. Le négoce maritime et le blocus continental, Lille, 
souhaiterait en savoir plus sur les «capitalistes » néerlandais et leurs stratégies d'exportation de capitaux, sur les possibilités ouvertes ou fermées par l'époque napoléonienne. Pouvait-on s'y enrichir et comment? Inversement, qu'en était-il des pauvres et des ouvriers? Johan Joor démontre que ce n'est pas chez eux qu'il faut rechercher les responsables des révoltes populaires. D'autres ont pourtant souligné combien la population avait souffert du chômage et de la misère. Mais ce sujet n'a pas encore fait l'objet de synthèses.

Les nouveaux objets, les nouveaux problèmes, les nouvelles approches incluent également l'histoire des concepts, sur le mode instauré par R. Koselleck ou par R. Reichardt. L'Institut d'histoire culturelle de l'Université d'Amsterdam y a consacré un programme de recherches communes et de publications; l'Institut Huizinga a rejoint l'initiative. Trois tomes sont parus, consacrés à la Liberté, à la Patrie et à la Civilisation (58); les deux prochains analyseront les concepts de Bourgeois et de République. Dans le prolongement de ces études conceptuelles, on peut évoquer de (trop) rares travaux sur l'histoire des idées politiques ou sur l'analyse des textes constitutionnels. Stephan Klein a ainsi renouvelé par le biais de la culture politique le livre fondateur de Leonard Leeb et fort bien analysé la spécificité du républicanisme patriote (59). Mais, alors que, progressivement sont publiés de vastes recueils de sources - juridiques, économiques, législatives - font encore défaut les études de fond sur les sources en question. L'on connaît fort peu par exemple les discussions parlementaires sur les articles de la constitution, qu'ils portent sur les assemblées primaires, les procédures électorales, les inclusions et exclusions ou sur les principes de souveraineté populaire et de volonté générale. L'ouvrage ne manque donc pas pour les historiens ou philosophes politiques, puisqu'il s'agit d'analyser et de comparer pas moins d'une dizaine de projets constitutionnels (si l'on inclut ceux réalisés par les patriotes et les exilés bataves), afin de voir ce qu'il en est au juste du républicanisme néerlandais et en quoi il se sépare ou non de la démocratie moderne (60).

(58) Vaderland, (éd. N.C.F. van Sas), Amsterdam, 1999. Vrijheid, (éd. E. O. G. Haitsma Mulier et W. VelemA), Amsterdam, 1999. Beschaving, (éd. P. DEN BOER), Amsterdam, 2001.

(59) S. KLEIN, Patriots Republikanisme. Politieke cultuur in Nederland 1766-1787, Amsterdam, 1995. I. L. Leeb, The ideological origins of the Batavian Revolution, La Hague, 1973. Voir aussi les études du grand historien E. H. KoSSMANN, Politieke theorie en geschiedenis, (plusieurs articles en anglais) Amsterdam, 1987.

(60) J. ROSENDAAL a présenté au colloque de Rome de 1998 un article sur divers projets inconnus. "Comment faire la révolution? Les projets révolutionnaires et leur réalisation aux Pays-Bas, 1786-1798 " (à paraître). Voir aussi S. KLEIN et J. ROSENDAAL, « Democratie in context. Nieuwe perspectieven op het Leids Ontwerp ", De Achttiende Eeuw, n²6, 1994, pp. 71-100. Notons encore De Eeuw van de grondwet: grondwet en politiek in Nederland 1798-1917, (éd. N. C. F. VAN SAS et H. TE Velde), Deventer, 1998. L'accent se pose ici surtout sur la constitution de 1848. Sur l'égalité et le bonheur, N. C. F. VAN SAS, « Begrippen in het constitutionele debat. Over Gelijkheid en Geluk», in Grondwetgeving 1795-1806, op. cit., pp. 51-64. 
Dans ce cadre, des études pourraient être entreprises sur les influences auxquelles ont été soumises les hommes de l'époque. Trop souvent est signalé combien les Bataves ont réactualisé un passé mythique, avant d'introduire malgré tout des nouveautés. Les Révolutions française et batave n'ont-elles pas précisément modifié les références? La mise en place de la Constitution de 1798 n'a-t-elle pas été ressentie comme un moment fondateur (61)? Enfin, les références à l'Antiquité ou au Siècle d'Or ne sont-elles pas avant tout formelles et l'inspiration ne découle-t-elle pas de modèles moins diserts, tels que la révolution américaine, les révolutions anglaises, et, sans nul doute, la Révolution française (62) ? Une analyse exhaustive de ces influences, et, des emprunts ou rejets auxquels elles ont donné lieu contribuerait sans doute à mieux préciser encore l'originalité de cette période mouvementée de l'histoire des Pays-Bas.

\section{Les résultats}

Si les synthèses et les monographies politiques sont rares; si plusieurs objets ci-dessus évoqués demandent encore à être traités, l'ensemble des travaux effectués au cours de la dernière décennie dessine une carte nuancée de ce que fut la République batave. Les Lumières, la révolution des patriotes de 1780-1787 et la révolution de 1795-1798 y figurent sous leurs traits spécifiques, marquées au sceau de la religion éclairée et rationnelle, d'une religion qui n'exclut ni le progrès, ni l'utilité et qui envisage comme sa tâche propre l'éducation du peuple (63). Spécifique aussi, l'État hollandais avec ses faiblesses, sa modération et son incapacité à rogner efficacement les prérogatives des provinces et des villes. Instruits par les expériences traumatisantes de leurs alliés, les Bataves comprennent qu'il ne faut point trop presser les choses; leur structure politique les a accoutumés du reste à des lenteurs inconnues en France, qui permettent de mieux appréhender leur rapport différent au temps. Dans ce contexte, le roi Louis ne parvint pas à imposer une centralisation universitaire et scientifique durable (64). Les anciennes structures se maintenaient discrètement, tout comme les coutu-

(61) Acceptée en avril 1798, il est vrai qu'elle est modifiée en 1801 sur les instances de Bonaparte; ensuite en 1805, en 1806 et en 1810, année de l'annexion de la Hollande à la France. Mais raison de plus pour qu'elle devienne, à l'instar de la Constitution de 1793, une référence fondatrice pour les républicains véritables. L'a-t-elle été?

(62) J. W. SCHULTE NORDHOLT, Voorbeeld in de verte. De invloed van de Amerikaanse revolutie in Nederland, Baarn, 1979. Voir le livre cité de S. KLEIN, qui démontre bien les influences anglo-saxonnes, voire rousseauistes ou son article dans Remous révolutionnaires, op. cit., pp. 25-33.

(63) M. C. JACOB, "Radicalism in the Dutch Enlightenment", in The Dutch Republic in the Eighteenth Century. Decline, Enlightenment and Revolution, op. cit., pp. 224-240. W. FrIJHOFF, «The Dutch Enlightenment and the Creation of Popular Culture », ibid., pp. 292-307.

(64) W. W. MUNHARDT, «Les sciences en révolution. Une affaire d'État », in Remous révolutionnaires, op. cit., pp. 200-219. Les savants hollandais s'opposèrent entre autres à la création d'une École polytechnique ; un Institut royal des Sciences fut créé, mais après bien des batailles. 
mes. Mais, du coup, la révolution batave ne provoqua pas une contre-révolution violente.

Des violences, il n'y en eut guère en effet. Les études locales le démontrent et révèlent en outre que les coalitions étaient fluctuantes et les divisions non insurmontables, d'autant que la répression fut fort modérée - tant en 1787 qu'en 1795. En 1799, le ministre de l'Éducation nationale, Van der Palm, appelle ainsi à la réconciliation nationale; il est rejoint en 1800 par Schimmelpenninck, ambassadeur de la République batave à Paris; à partir de 1801 , orangistes et révolutionnaires travaillent de concert à l'Assemblée nationale; et, en 1813, tous (y compris les « jacobins » bataves) acclament le retour du prince d'Orange. Il est vrai, et plusieurs travaux le démontrent, tels ceux de Wyger Velema, qu'orangistes et révolutionnaires étaient pour une grande part hommes des Lumières. S'ils se séparaient sur le rôle à conférer au stadhouder et sur le problème de la souveraineté, ils se retrouvaient dans leur désir de réformes et dans leur aspiration à l'unité - de même que dans leur mépris pour la «populace». Aussi les orangistes ne peuvent-ils en aucun cas être confondus avec des réactionnaires et les révolutionnaires avec des Jacobins. Bien des nuances étaient présentes chez les uns comme chez les autres, on l'a entrevu, mais, surtout, ces nuances s'estompèrent dès que fut menacée l'existence même de la patrie, ce qui fut le cas après l'incorporation du royaume de Hollande à l'empire français. En 1813, orangistes et révolutionnaires unirent leurs forces pour chasser les occupants étrangers.

Bien que le rôle des élites dans la révolution ait été important à tous les niveaux, ce ne veut point dire que la participation populaire ait été réduite à une peau de chagrin. Le peuple a pour une part été politisé durant la révolution patriote par le biais des sociétés, de la presse politique, des images et des pamphlets, mais encore par les actions des corporations, les initiatives locales et les élections municipales; la République batave lui permit de prendre une part plus grande encore aux élections et d'investir les assemblées de quartier et les assemblées primaires, tandis que des artisans, des boutiquiers, des marchands faisaient leur entrée au conseil municipal. Plus encore qu'en France, où, sous le Directoire, l'enseignement devint scientifique et élitaire, les législateurs bataves se flattaient d'instruire et de former les masses incultes (65). La pédagogie dix-huitiémiste s'imposa, tout comme l'idée de régénération, qui, faute de moyens et de persévérance, ne parvint pas néanmoins à prédominer. Quant aux femmes, régénérées ou non, elles subirent le sort que leur avaient réservé les patriotes français : à peine sorties de leur foyer, elles durent y retourner et se consacrer à des tâches

(65) Voir à ce sujet, G. CUVIER et F. J. NOĚL, Rapport sur les établissements d'instruction publique en Hollande, Paris, 1811, où les auteurs témoignent de leur admiration pour le modèle batave. Et W. W. MIJNHARDT, article cité note précédente. 
mieux accordées avec leur sensibilité. Dans un camp comme dans l'autre, il y en eut cependant pour défier les lois. Kaatje Mossel, femme du peuple orangiste, à l'origine des troubles de Rotterdam de 1784, ou Aletta Hulshoff, auteur d'un pamphlet anti-napoléonien : à quelques décennies de distance, toutes deux se retrouvèrent en prison. Betje Wolf et Aagje Deken, écrivains célèbres et « véritables amies de la Liberté » adoptèrent une autre stratégie; elles inondèrent l'espace public d'écrits moraux et didactiques et accablèrent l'Assemblée d'adresses incisives. Les injonctions des pouvoirs n'eurent donc pas toujours raison de l'opiniâtreté féminine; jusqu'en 1800-1808, la politisation touchait jusqu'aux minorités, fussent-elles exclues de l'arène officielle (66).

Enfin, les Bataves ont beau invoquer leur glorieux passé, contrairement aux patriotes, ils ne cherchent donc plus à restaurer leur constitution originelle de 1579, mais à fonder une tradition nouvelle, inspirée de l'exemple tant américain que français et harmonisée avec ce qu'ils jugent être leur caractère national. La véritable rupture révolutionnaire, ils la vivent en 1798. C'est celle qui détruit à jamais l'ancienne fédération des ProvincesUnies - point sur lequel ils font œuvre plus radicale qu'en France, puisque la Convention parachève bon gré mal gré l'œuvre entreprise depuis Louis XIV. Et, de ce fait, la révolution batave est bien une rupture - à la fois brutale et modérée, en ce sens que des mesures «radicales» sont certes décrétées, mais elles mettront plus de six ans pour entrer réellement en vigueur. Les législateurs comprennent qu'ils ne peuvent contraindre les sept provinces par la force et la violence (67) - à l'exception des radicaux de janvier-juin 1798 qui épurent les assemblées primaires, mais n'exercent pas une répression brutale. La tradition n'est pas morte. Les protestations régionales et locales s'expriment par le biais de pétitions et l'État impose ses lois par des décrets, en espérant qu'ils seront à la longue appliqués. La fameuse modération révolutionnaire batave découle sans nul doute de l'ancienne structure politique et de la culture religieuse, mais aussi de l'expérience vécue en France entre 1789 et 1794 et de la conjoncture financière désastreuse qui repousse à l'arrière-plan les beaux projets de régénération et de démocratisation.

Alors la révolution batave, un modèle ? C'est bien ainsi qu'elle apparaît actuellement dans les manuels scolaires et les synthèses universitaires: comme la source de l'État-Nation moderne, réalisé sans effusion de sang, tout juste avec quelques dettes, tandis que la période et l'influence française reprennent leur place de second rang. La Constitution de 1798 est bel et

(66) M. EVERARD, “Vrouwen voor't vaderland : burgeres Van der Meer en de Bataafse politiek », Holland, vol. 31, 1999, pp. 272-281.

(67) Notons que les sept provinces, avec la révolution de 1795, sont devenues neuf. Celles de Drenthe et du Brabant ont alors été admises aux États-Généraux, ce qui n'était pas le cas jusque-là. 
bien néerlandaise, ainsi que la centralisation et l'État unitaire qui en découlent. Tout cela n'a pas été imposé d'en haut, mais souhaité à divers niveaux par des hommes enfin devenus conscients que leur nostalgie du Siècle d'Or ne le ressusciterait pas.

Annie Jourdan Université d'Amsterdam Lijnbaansgracht 348 N. L. - 1017 XB Amsterdam

Joost RosENDAAL Université de Nimègue Surinameweg 15 N. L. -6524 SW Nijmegen 